\title{
Radial propagation of structures in drift wave turbulence
}

\author{
T. Windisch,* O. Grulke, and T. Klinger \\ Max-Planck-Institute for Plasma Physics, \\ EURATOM Association, D-17491 Greifswald, Germany
}

(Dated: October 26, 2006)

\begin{abstract}
The formation and propagation of spatiotemporal fluctuation structures in weakly developed drift-wave turbulence in a linearly magnetized helicon device is investigated. Turbulent density fluctuations in the far edge plasma display an intermittent character with large-amplitude positive density bursts. Their peak amplitudes correspond to the time-averaged density in the maximum radial plasma pressure gradient. The conditional average technique $(\mathrm{CA})$ is applied to reconstruct the dynamics of turbulent coherent structures in the azimuthal plane. The formation of turbulent structures is closely linked to a quasi-coherent $m=1$ drift wave mode, which is generally observed in the radial density gradient region in the weakly developed turbulent state. It is demonstrated that every positive high amplitude density burst in the plasma edge is due to the radial propagation of a turbulent structure. The typical scale size of the turbulent structures is $4 \rho_{s}$ and their lifetime exceeds the eddy turnover time by orders of magnitude, thereby characterizing them as coherent structures. Although the turbulent structures propagate mainly azimuthally in direction of the $\mathrm{E} \times \mathrm{B}$-drift they are observed to have a radial velocity, which is typically $10 \%$ of the ion sound speed.
\end{abstract}

PACS numbers: $52.35 . \mathrm{Kt}, 52.35 . \mathrm{Ra}, 52.25 . \mathrm{Xz}$

*Electronic address: thomas.windisch@ipp.mpg.de 


\section{INTRODUCTION}

Motivated by the observation of particle and energy transport perpendicular to the magnetic field of fusion devices, the fluctuation induced cross-field plasma flow into the far scrape-off layer (SOL) has been the subject of attention during the last two decades [1-3]. Owing to fluctuation-induced transport, one observes the formation of a pronounced shoulder in the radial density profile and enhanced recycling of plasma particles at the first wall [4]. This has a strong impact on divertor efficiency and might affect key reactor issues like helium "ash" removal.

A characteristic feature of such a convective particle flux in the SOL are intermittent density bursts, characterized by a non-Gaussian amplitude probability distribution function (PDF). These density bursts can be ascribed to large amplitude spatiotemporal turbulent structures called blobs, that propagate radially outwards through the SOL with a velocity of typically less than one tenth of the ion sound speed [3] and eventually reach the first wall. Blobs are localized in the poloidal plane but they are elongated along the magnetic field lines, thereby forming extended filaments with $k_{\perp} \gg k_{\|}$[5]. It was recently realized that blobs contribute considerably to the radial transport in the SOL [6]. Common model descriptions addressing the radial blob propagation in the SOL of tokamaks usually rely on the curvature of the magnetic field, which causes either polarization of the blobs [7] or gives rise to electrostatic interchange dynamics [8]. However, intermittent density fluctuations in the plasma edge and the formation and radial propagation of spatiotemporal structures have also been observed in linear devices with homogeneous magnetic field geometry $[9,10]$. The universality of intermittent convective transport, independent of the magnetic field geometry, was underlined in Ref. [11] after a comparison of statistical features of density fluctuations in the edge plasma of toroidal and linear devices. A neutral wind model [12] was suggested to explain the radial propagation of turbulent structures in partially ionized plasmas in linear devices. The polarization of the turbulent structure is achieved in the neutral wind model by friction between ions and neutrals.

In the present paper we characterize the formation and propagation of turbulent structures from weakly developed drift wave turbulence in the linear VINETA device. Crossconditional techniques are used to reconstruct statistically the spatiotemporal dynamics of the fluctuations. The paper is organized as follows: The experimental setup and the statisti- 


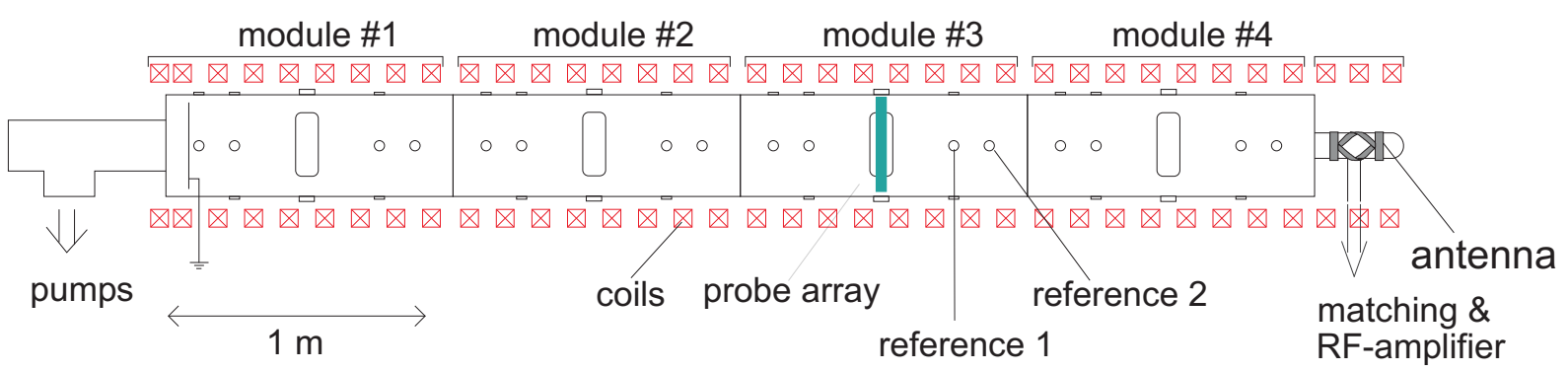

FIG. 1: Schematic drawing of the VINETA device. The axial locations of the probe diagnostics used for the present studies are explicitly shown.

cal data analysis tools are outlined in Sec. II. Section III deals with the temporal evolution of density fluctuations across the radial plasma density profile. The experimental results of formation and propagation of spatiotemporal structures are characterized in Section IV and are summarized in Sec. V.

\section{EXPERIMENTAL SETUP AND DATA ANALYSIS}

The experiments were carried out in the linearly magnetized helicon device VINETA, schematically depicted in Fig. 1. It consists of four separate stainless steel vessels with a diameter of $0.4 \mathrm{~m}$ and has a total length of $4.5 \mathrm{~m}$.

The vacuum vessel is immersed in a set of 36 magnetic field coils, which provide a homogeneous magnetic field with induction $B \leq 0.1 \mathrm{~T}$. Plasma is produced by a conventional helicon source [13] with a helical $m=+1$ antenna, placed around a cylindrical glass vacuum extension of $0.1 \mathrm{~m}$ diameter and $0.5 \mathrm{~m}$ length. The antenna is coupled to a radio frequency (rf)-source $\left(f_{r f}=13.56 \mathrm{MHz}, P_{r f} \leq 5 \mathrm{~kW}\right)$ via a capacitive matching network. It is a general feature of helicon discharges to produce a rather dense plasma at low electron temperatures [14]. The present investigations are done in an Argon plasma with a neutral gas pressure of $p=0.1 \mathrm{~Pa}$, a magnetic field of $B=60 \mathrm{mT}$, and a rf power of $P_{r f}=1.8 \mathrm{~kW}$. Typical plasma parameters and the temporal and spatial scales are compiled in Tab. I. Due to the high plasma density and low electron temperature the helicon plasma in VINETA is characterized by a fairly high Coulomb collision frequency $\nu_{e i} / \Omega_{c i} \approx 100$ and high electron-neutral collision frequency $\nu_{e n} / \Omega_{c i} \approx 10$.

Time-averaged radial plasma density and potential profiles are measured with Langmuir 
TABLE I: Typical plasma parameters.

\begin{tabular}{lr}
\hline \hline Plasma parameter & Typical value \\
\hline Peak electron density $n$ & $1.2 \cdot 10^{18} \mathrm{~m}^{-3}$ \\
Electron temperature $T_{e}$ & $3 \mathrm{eV}$ \\
Ion temperature $T_{i}$ & $0.2 \mathrm{eV}$ \\
Ion gyro frequency $\Omega_{c i}$ & $1.48 \cdot 10^{5} \mathrm{rad} / \mathrm{s}$ \\
Ion sound speed $c_{s}$ & $2.7 \mathrm{~km} / \mathrm{s}$ \\
Ion gyro radius $r_{c i}$ & $6.6 \mathrm{~mm}$ \\
Drift-scale $\rho_{s}$ & $18 \mathrm{~mm}$ \\
Density gradient length $L_{\perp}^{-1}=|\nabla n| / n$ & $51 \mathrm{~m}^{-1}$ \\
\hline \hline
\end{tabular}

probes, which are compensated against rf-fluctuations [15]. The measured density profiles are calibrated versus the line-integrated density as measured with a $160 \mathrm{GHz}$ interferometer. Radial profiles of the plasma density and the plasma potential are shown in Fig. 2. Both the plasma density and plasma potential profile have a Gaussian shape but with different $1 / e$-folding lengths $(46 \mathrm{~mm}$ for the plasma density and $169 \mathrm{~mm}$ for the plasma potential profile). Two-dimensional measurements in the azimuthal plane reveal that the profiles are azimuthally symmetric.

The modulus of the azimuthal $\mathrm{E} \times \mathrm{B}$-drift $v_{\mathrm{E} \times \mathrm{B}} \sim-\nabla \phi / B$ and the electron diamagnetic drift $v_{\mathrm{d}, \mathrm{e}} \sim-\nabla \ln n / B$ perpendicular to the magnetic field, are calculated from the exponential fits to the time-averaged radial profiles. The result is shown in Fig. 3. The electron diamagnetic drift velocity $v_{\mathrm{d}, \mathrm{e}}$ has a maximum value of $2.1 \mathrm{~km} / \mathrm{s}$ in the plasma density gradient region at $r=61 \mathrm{~mm}$ and decreases towards the plasma edge and center. Due to the different radial scale lengths of the density and potential profiles the electron diamagnetic drift dominates over the $\mathrm{E} \times \mathrm{B}$-drift in the bulk plasma. At a certain radial position $(r=82 \mathrm{~mm})$ the $\mathrm{E} \times \mathrm{B}$-drift exactly balances the electron diamagnetic drift, such that the effective drift velocity is zero (note that both drift-velocities have opposite sign). In the far plasma edge, only the $\mathrm{E} \times \mathrm{B}$-drift contributes significantly to the total azimuthal drift. The $\mathrm{E} \times \mathrm{B}$-drift velocity has a maximum value of $1.5 \mathrm{~km} / \mathrm{s}$ at $r=124 \mathrm{~mm}$ and decreases towards smaller and larger radii.

As has been shown by Schröder et al. [16] the governing instability in the VINETA device 


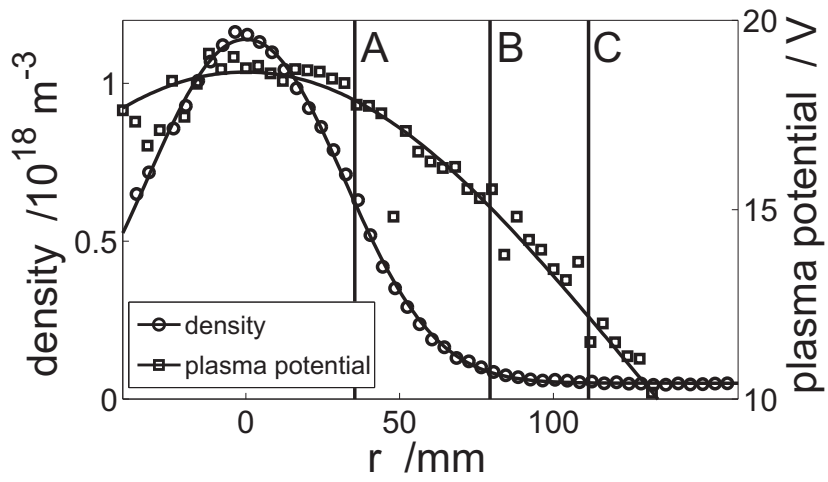

FIG. 2: Time-averaged radial plasma density and plasma potential profile (the solid lines are Gauss fits to the data).

is the drift wave instability. Drift waves satisfy the low-frequency condition $\left(\omega \ll \Omega_{c i}\right)$ and are driven by the free energy provided by the pressure gradient perpendicular to the magnetic field. Coherent drift wave modes with azimuthal mode numbers int the range $m=1-9$ and frequencies in the range $\omega / 2 \pi=1-10 \mathrm{kHz}$ are observed in the VINETA device. An important parameter for the destabilization of drift waves is the ratio of the perpendicular density gradient length to the drift scale, $L_{\perp} / \rho_{s}$. Changes of the magnetic field effect predominantly the drift wave scale $\rho_{s}$ while the radial density and potential profiles remain essentially unchanged. The mode number of drift waves are observed in VINETA to increase with decreasing $\rho_{s}$, i.e. an increase of the magnetic field [16]. For high magnetic fields the plasma is in a weakly developed turbulent state.

Plasma density fluctuations are measured with uncompensated Langmuir probes, operated in the ion saturation current regime at a probe bias of $U=-100 \mathrm{~V}$ with respect to 
the grounded plasma vessel (electron temperature fluctuations are assumed to be negligible, such that $\tilde{I}_{\text {sat }} \sim \tilde{n}$ ). To gain insight into the spatiotemporal dynamics in the azimuthal plane we arranged 16 single probes as a vertical array perpendicular to the ambient magnetic field (cf. Fig. 4). The probes have a vertical spacing of $\Delta y=8 \mathrm{~mm} \approx \rho_{s} / 2$. The individual probes consist of a tungsten wire ( $0.1 \mathrm{~mm}$ diameter $)$ that is electrically insulated with a ceramic tube ( $0.6 \mathrm{~mm}$ diameter) except for the probe tip (3 $\mathrm{mm}$ length). The array is mounted on a computer controlled positioning system and can be radially moved across the entire plasma column. Time series of plasma density fluctuations are recorded with high temporal resolution (1.25 MHz sampling frequency), 12 bit amplitude resolution, and a record length of $128 \mathrm{k}$ samples.

Spatiotemporal structures are extracted by applying the conditional averaging (CA) method $[17,18]$, which is based on the statistical comparison of two simultaneously recorded time series at different spatial positions. One time series is recorded at a a fixed spatial position $\mathbf{r}_{\mathbf{0}}$ and acts as a reference signal $R\left(\mathbf{r}_{0}, t\right)$. The other time series is recorded simultaneously but at a different spatial position $\mathbf{r}_{0}+\mathrm{d} \mathbf{r}$ and is called the displaced signal $D\left(\mathbf{r}_{0}+\mathrm{d} \mathbf{r}, t\right)$. When the reference signal $R$ fulfills a pre-defined condition $p$ at a certain time instant $t_{i}$, a time interval of length $\Delta \tau$ centered around $t_{i}$ is extracted from both time series. If the condition is fulfilled $N$ times, the $N$ sub-time series are taken as statistically independent realizations. By ensemble averaging of the sub-time series the coherent part of the fluctuations is extracted while the incoherent part is suppressed. The conditional

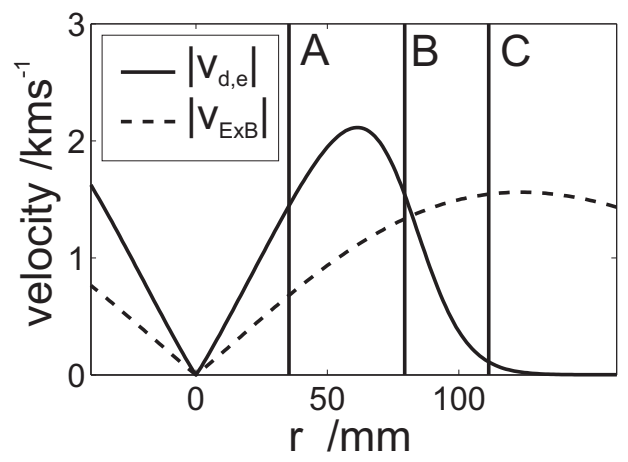

FIG. 3: Absolute $\mathrm{E} \times \mathrm{B}$ - and electron diamagnetic drift velocities as calculated from the time averaged plasma density and plasma potential profile (cf. also Fig. 2) 


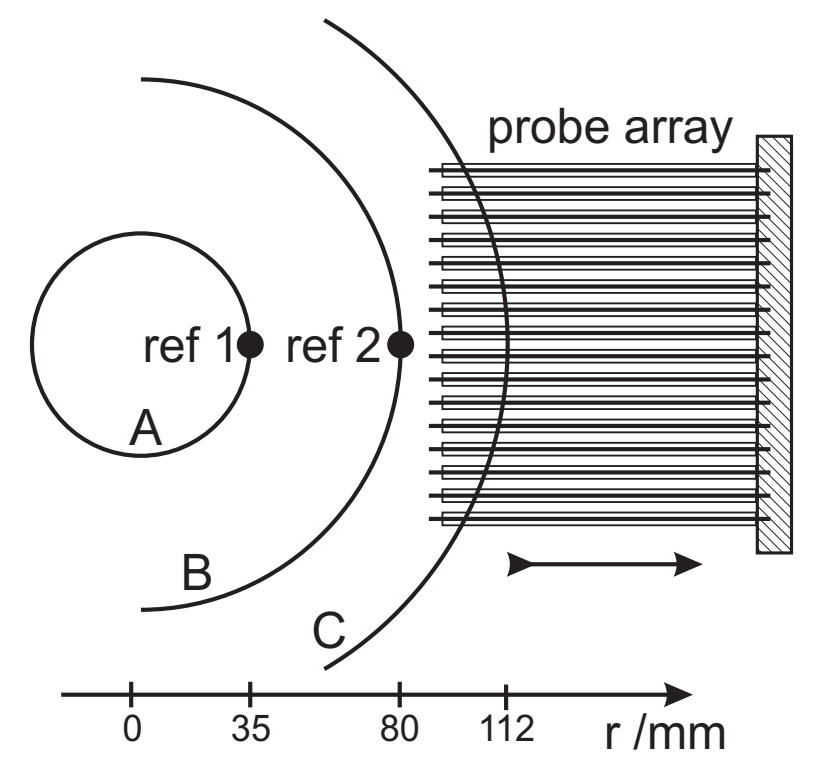

FIG. 4: Experimental setup for the spatiotemporal fluctuation diagnostics. For single-point density fluctuation measurements, three Langmuir probes at radial positions A,B and C are used. The spatiotemporal density fluctuations in the azimuthal plane are measured with a vertical probe array, that is moved across the plasma profile. The magnetic field is pointing out of the plane.

averaging procedure can be expressed as

$$
\langle R\rangle_{\mathrm{CA}}\left(\mathbf{r}_{0}+\mathrm{d} \mathbf{r}, \tau\right)=\frac{1}{\mathrm{~N}} \sum_{\mathrm{i}=1}^{\mathrm{N}} \mathrm{D}_{\mathrm{i}}\left(\mathbf{r}_{0}+\mathrm{d} \mathbf{r}, \mathrm{t}_{\mathrm{i}}+\tau\right), \quad \tau \in[-\Delta \tau / 2, \Delta \tau / 2] .
$$

The condition $p$ is a free parameter and is chosen here as a threshold condition on the amplitude combined with a slope condition. Events that reach the predefined threshold amplitude within an amplitude interval of $p \pm 5 \%$ are detected at falling slope. The time interval is chosen to $\Delta \tau= \pm 50 \mu \mathrm{s}$.

In the next section single-point measurements of density fluctuations are compared at three radial positions are, in the following referred to as A, B and C. These positions are indicated in Figs. 2, 3, and 4. Position $\mathrm{A}$ is in the radial density gradient region, where the electron diamagnetic drift dominates. Position B is in the plasma edge, where the density drops down to $7 \%$ of the peak density and the effective azimuthal drift frequency almost vanishes. In the far plasma edge, at position $\mathrm{C}$, the time-averaged density further decreases to $4 \%$ of the peak density and the azimuthal drift is governed by the $\mathrm{E} \times \mathrm{B}$-velocity. We reconstruct the spatiotemporal dynamics of density fluctuations in the azimuthal plane by applying the CA method as follows: We use the density fluctuations measured with 
Pos.
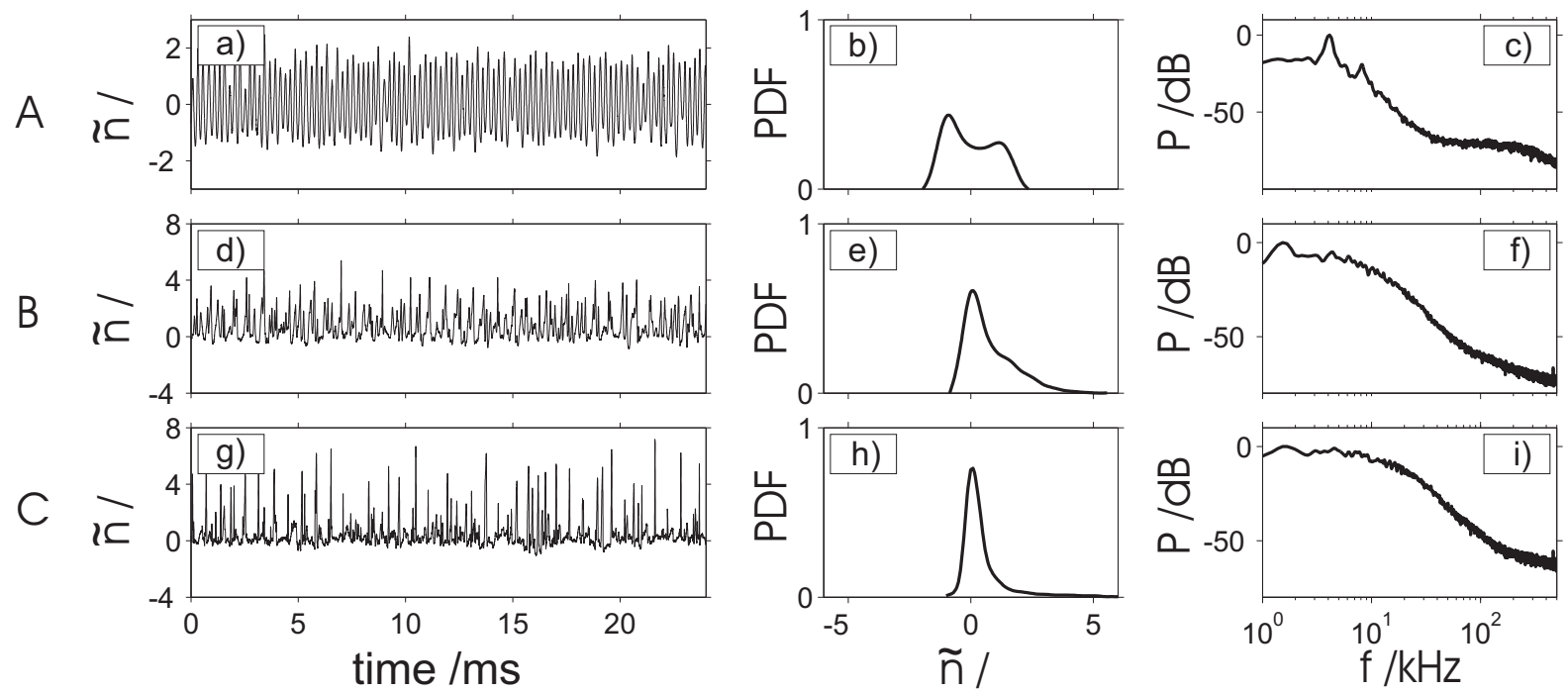

FIG. 5: Time series of density fluctuations, probability distribution function (PDF) and frequency power spectra at radial positions A (a-c), B (d-f) and C (g-i) as indicated in Fig. 2, respectively.

Langmuir probes at the positions $\mathrm{A}$ and $\mathrm{B}$ as the reference signal $R\left(\mathbf{r}_{0}, t\right)$. Density fluctuation time series are recorded from the plasma edge towards the plasma core with the movable probe array and are taken as the displaced signal $D\left(\mathbf{r}_{0}+\mathrm{d} \mathbf{r}, t\right)$. The axial distance along the magnetic field between the reference probes and the probe array is $\Delta z=42 \mathrm{~cm}$ and $\Delta z=29 \mathrm{~cm}$ upstream, respectively (cf. Fig. 1).

\section{TEMPORAL EVOLUTION OF DENSITY FLUCTUATIONS}

For characterization of the temporal evolution of turbulent density fluctuations in radial direction, single-point measurements of density fluctuations at positions $\mathrm{A}, \mathrm{B}$, and $\mathrm{C}$ are depicted in Fig. 5. Shown are the density fluctuation time series normalized to standard deviation $\tilde{n} / \sigma$, the respective probability distribution function (PDF), and frequency power spectra $P$. The character of the plasma density fluctuations changes significantly across the radial plasma density profile: In the density gradient (position A, Fig. 5.a-c), coherent density fluctuations with relatively low magnitude $(\tilde{n} / \sigma \leq 2)$ are observed. This is confirmed by the frequency power spectrum, that peaks at a frequency of $4.1 \mathrm{kHz}$. This peak frequency is smaller than the electron diamagnetic drift-frequency at the radial position A $\left(f_{\mathrm{d}, \mathrm{e}}=v_{\mathrm{d}, \mathrm{e}} / 2 \pi r=6.5 \mathrm{kHz}\right)$. For higher frequencies $(f>8 \mathrm{kHz})$ in the power spectrum a 


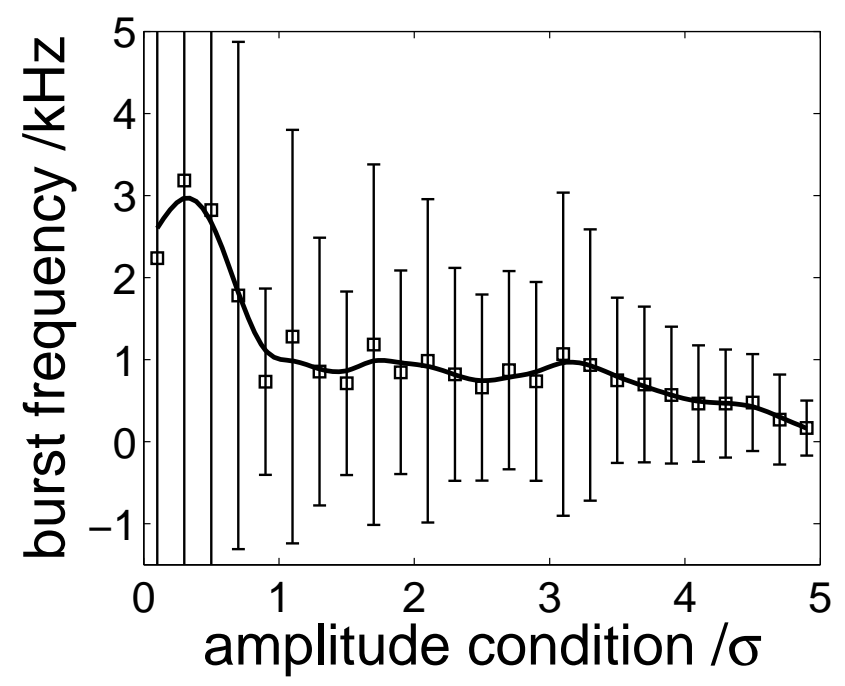

FIG. 6: Frequency of positive density bursts in the far plasma edge (pos. C in Fig. 2).

power-law decrease is observed. The PDF is symmetric and double-humped as expected for coherent fluctuations. The density fluctuations in the plasma edge (pos. B, Fig. 5.d) have an intermittent character with positive density bursts of peak amplitudes $\tilde{n} / \sigma \sim 5$. The fluctuations are asymmetric, which results in a peaked PDF having a pronounced positive tail. This is quantified by the moments of the PDF skewness $S=1.1$ and kurtosis $K=1$. In comparison with position A (Fig. 5.c) the frequency power spectrum is broader and no pronounced peak at $f=4.1 \mathrm{kHz}$ is observed. However, the occurrence of the positive density bursts is not completely random in time but is centered around a characteristic frequency, which results in a broadened peak at $f=1.3 \mathrm{kHz}$ (c.f. Fig. 5.f). The intermittent character of density fluctuations further increases towards the far edge (pos. C, Fig. 5.g-i), where the time-averaged density is already low. Positive density bursts are observed with normalized amplitudes up to $\tilde{n} / \sigma \sim 7$. The maximum burst densities correspond to the density fluctuation amplitude in the maximum radial gradient region, where the relative fluctuation amplitude $\tilde{n} / n_{0}$ is typically $10 \%$. The PDF in the far edge is asymmetric and peaked with $S=2.9$ and $K=11$. The frequency power spectrum shows a power law decrease for frequencies $f \geq 10 \mathrm{kHz}$, no pronounced peaks are observed.

The temporal distribution of events in the density fluctuation time series at the plasma edge (pos. C) is determined by detecting the time instant of each fluctuation peak which meets a predefined amplitude condition (an amplitude window of $5 \%$ is imposed to increase the number of detected events). Averaging over the time delay between two consecutive 
events then yields an event "frequency" for the individual amplitude condition. Fig. 6 shows the obtained burst frequency for increasing amplitude condition $p=1-5 \sigma$. The error bars represent the standard deviation of the so obtained frequencies. It is observed that the occurrence of bursts for various amplitudes is not completely random but corresponds to a characteristic frequency range of $0.5-1 \mathrm{kHz}$ for bursts with amplitudes $p>1 \sigma$. This is particularly evident for the largest amplitude bursts, for which the error bars are mainly given by the relatively small numbers of detected events (only 40 detected events for an amplitude condition of $p=3 \sigma$ ). For smaller amplitudes $p \leq 1 \sigma$ noise-like small amplitude fluctuations contribute significantly to the obtained frequencies, which leads to the large error bars.

\section{SPATIOTEMPORAL EVOLUTION OF DENSITY FLUCTUATIONS}

As pointed out in Sec. III, the amplitudes of the positive density bursts in the far plasma edge (pos. C) are comparable to the time-averaged density in the maximum radial density gradient region. This finding suggests that the density bursts in the far plasma edge originate from the radial density gradient region. To investigate the radial fluctuationinduced convective particle flux across the axial magnetic field, measurements of density fluctuations in the entire azimuthal cross-section have been done. The reconstruction of the spatiotemporal density fluctuations in the azimuthal plane is done by applying the CA method, as described in Sec. II, for the two reference positions A and B. The reference probe is located in the density gradient region (pos. A, indicated by the black dot) and the result of the CA analysis is shown in Fig. 7 for two different time lags $\tau$.

For both time lags a coherent $m=1$ drift wave mode dominates the coherent part of the density fluctuations. The peak fluctuation amplitudes of the mode are located in the maximum gradient region of the (time-averaged) density profile. The mode propagates purely azimuthally in direction of the electron diamagnetic drift (clockwise in the representation of Fig. 7). No radial component for the mode propagation is observed. The observed phase velocity of the mode is $v_{\varphi} \approx 1 \mathrm{~km} / \mathrm{s}$, which is a factor of 1.2 smaller than the electron diamagnetic velocity minus the $\mathrm{E} \times \mathrm{B}$ velocity. For the drift wave dispersion relation derived from the Hasegawa-Mima model [19],

$$
\omega=\omega^{\star} /\left(1+\left(k_{\perp} \rho_{s}\right)^{2}\right),
$$


where $\omega^{\star}=v_{\mathrm{d}, \mathrm{e}} / r$ is the electron diamagnetic drift frequency, for the observed $m=1$ mode $k_{\perp} \rho_{s}=0.56$. This yields a mode frequency of $\omega_{(\mathrm{m}=1)}=\omega^{\star} / 1.3$, which is in excellent agreement with our observations.

In addition to the drift mode structure, another conditionally averaged density structure is observed in Fig. 7 (time lag $\tau=-28 \mu \mathrm{s}$ ). A coherent plasma density fluctuation structure peels off the drift wave mode and propagates in radial direction, while the mode itself propagates further in azimuthal direction. The formation of this coherent turbulent structure occurs in the density gradient region and, since the reference signal is located in the region where the drift mode exists, its phase is coherent with the drift mode itself. The turbulent structure is azimuthally distorted with an azimuthal extent of $\Delta y \approx 60 \mathrm{~mm}$ and a radial extent of $\Delta x \approx 50 \mathrm{~mm}$ Its peak amplitude is $0.8 \sigma$. We note that the formation process of the coherent turbulent structure occurs in a region where a strong radial velocity shear exists (cf. Fig. 3). At radial positions $r \leq 90 \mathrm{~mm}$ the azimuthal drift velocity is no longer dominated by the electron diamagnetic drift but by the $\mathrm{E} \times \mathrm{B}$-drift, which has the opposite direction. To investigate specifically the spatiotemporal dynamics of the turbulent structure, the CA procedure is applied to data with the reference signal recorded at position B (cf. in Fig. 2). The resulting CA amplitude in the azimuthal plane is shown for four different time lags $\tau$ in Fig. 8. The position of the reference probe is again indicated by a black

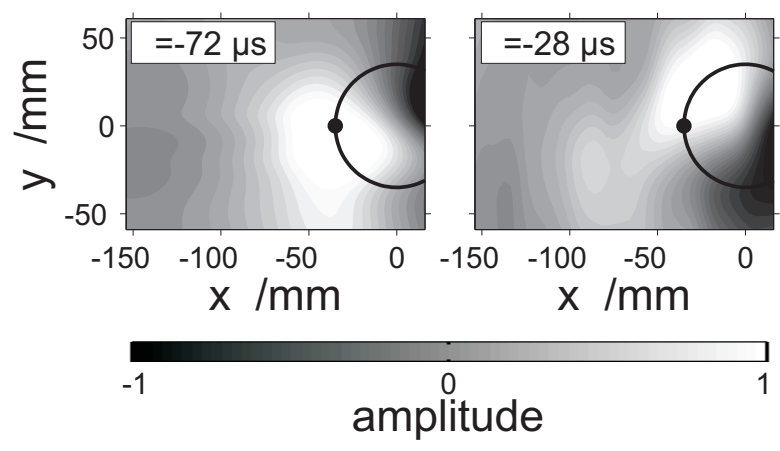

FIG. 7: Conditional average of density fluctuations for two different time lags $\tau$ in the azimuthal plane. The reference probe is located in the density gradient region (indicated by a black dot) and the amplitude condition was chosen to be $p=\sigma / 2$. For convenience, the CA amplitude is normalized to standard deviation and the color axis was set to $1 \sigma$. The black circle denotes a purely azimuthal propagation of the $m=1$ drift-wave mode. 


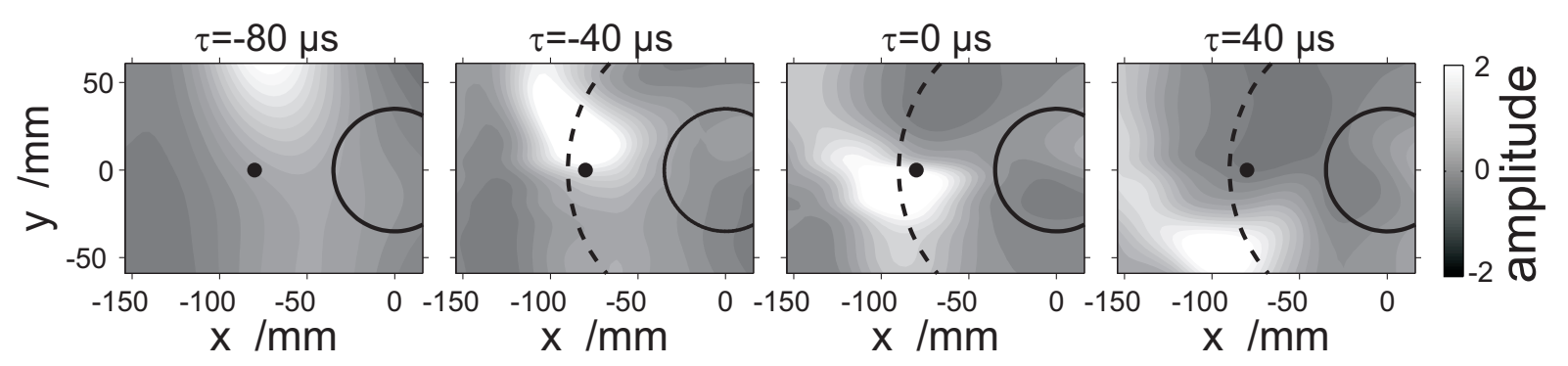

FIG. 8: Conditional average of density fluctuations for four different time lags $\tau$ in the azimuthal plane. The amplitude condition for the reference probe which is located in the plasma edge $(\mathrm{r}=-80$ $\mathrm{mm}$ ) was chosen to $\mathrm{p}=3 \sigma$. For convenience, the $\mathrm{CA}$ amplitude is normalized to standard deviation and the color axis was set to $2 \sigma$. The black solid circle corresponds with the position of reference probe one, identical with Fig.7. The black dashed indicates the trajectory of the coherent structure if it would propagate purely azimuthally.

dot. The CA amplitude condition is now chosen to be $p=3 \sigma$ in order to extract the positive high-amplitude density fluctuations. A propagating coherent turbulent structure is clearly observed in the plasma edge. Its amplitude exceeds by far the time averaged density $n_{0}$ with $n_{\text {burst }} \approx 6 n_{0}$. Cross-correlation analysis yields a maximum correlation length of $l_{\text {corr,pol }} \approx 70 \mathrm{~mm}$ in the azimuthal direction and $l_{\text {corr,rad }} \approx 50 \mathrm{~mm}$ in the radial direction. In contrast to the coherent $m=1$ mode (Fig. 7), the structure propagates in azimuthal direction, but opposite i.e., the direction of the $\mathrm{E} \times \mathrm{B}$ drift. However, its trajectory is not anymore purely azimuthal (indicated by the dashed circle in Fig. 8) but has a significant radial component. The azimuthal and radial propagation velocities are analyzed in detail by tracking the turbulent structure over the two-dimensional domain for time lags $\tau=-40 \ldots 40 \mu \mathrm{s}$ in steps of $4 \mu \mathrm{s}$. Only the high-amplitude area of the structure with amplitudes exceeding 80\% the peak amplitude is considered. The center-of-mass position of this area is tracked through the domain and the resulting positions are decomposed in azimuthal angle and radial displacement. This, together with the time difference yield by the CA procedure, provides for each time instant the azimuthal and radial structure velocity, as shown in Fig. 9. In azimuthal direction (Fig. 9.a), a constant velocity of $v_{\text {pol }}=760 \mathrm{~m} / \mathrm{s}$ is found for all analyzed time instants. This corresponds to an azimuthal rotation frequency of $f_{\mathrm{pol}}=1.35 \mathrm{kHz}$. This frequency is in good agreement with the burst frequency of the high- 
amplitude density fluctuations (Fig. 6). The azimuthal velocity of the turbulent structure is $50 \%$ of the local $\mathrm{E} \times \mathrm{B}$-velocity $\left(v_{\mathrm{E} \times \mathrm{B}}=1.42 \mathrm{~km} / \mathrm{s}\right)$. A possible explanation for this deviation is a non-negligible contribution of the electron diamagnetic drift-velocity, which would lower the effective azimuthal velocity to $v_{\text {pol,eff }}=572 \mathrm{~m} / \mathrm{s}$. Although the scatter in the obtained radial velocities (Fig. 9.b) is larger, a relatively constant radial structure velocity of $v_{\text {rad }}=230 \mathrm{~m} / \mathrm{s}$ is found, which corresponds to $\approx 10 \% c_{s}$, where $c_{s}$ is the local ion sound speed.

Pictorially, the propagation of the turbulent structure can be described by a spiral motion in the azimuthal plane.

For the time lags shown in Fig. 8, a slight azimuthal velocity shear of the turbulent structure is observed in the far plasma edge $(x=-130 \mathrm{~mm})$. This can be explained by the radial evolution of the $\mathrm{E} \times \mathrm{B}$-drift in the far plasma edge (cf. Fig. 3), which peaks at $x=124 \mathrm{~mm}$ and decreases towards the plasma vessel. An analysis of the correlation time $\tau_{c}$ of the turbulent structure is not possible due to the spatial limitation of the measurement in the azimuthal plane. From the results shown in Fig. 8, only a lower limit of $\tau_{c}>150 \mu \mathrm{s}$ is estimated. The radial velocity of the turbulent structure $v_{\mathrm{rad}}=230 \mathrm{~m} / \mathrm{s}$ corresponds to a radial displacement of $\Delta r=17 \mathrm{~cm}$ during one azimuthal turn, such that every turbulent structure is detected only once.

If the fluctuation induced perpendicular particle flux $\tilde{\Gamma}_{\perp}^{\text {burst }}=<n_{\text {burst }} v_{\text {rad }}>$ caused by large-amplitude turbulent structures with $\tilde{n} / \sigma>3$ is compared to the parallel particle flux $\Gamma_{\|}=<n_{0} c_{s}>$ towards the end plate at $x=-90 \mathrm{~mm}$, one finds that the parallel flux still dominates by several orders of magnitude, $\tilde{\Gamma}_{\perp}^{\text {burst }} / \Gamma_{\|} \approx 6 \cdot 10^{-4}$, as expected for a linear device.

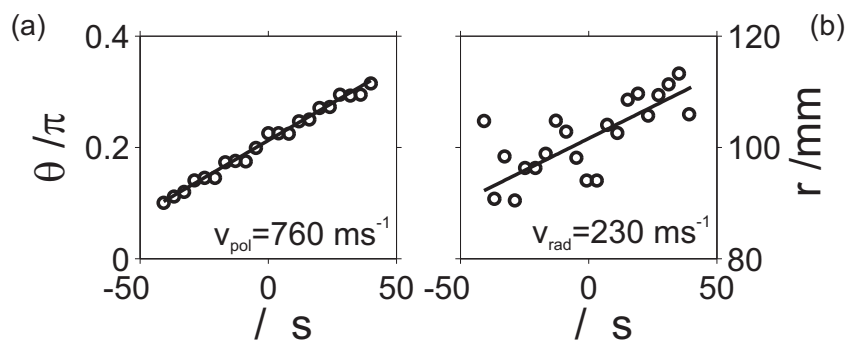

FIG. 9: Azimuthal (a) and radial (b) velocity of the turbulent coherent structure observed in Fig. 8. 


\section{SUMMARY AND DISCUSSION}

This paper investigates the formation and propagation of coherent turbulent structures in weakly developed drift wave turbulence in a linearly magnetized helicon device. The one-dimensional measurements (Sec. III) as well as the spatiotemporal measurements (Sec. IV) of plasma density fluctuations reveal the presence of a $m=1$ drift wave mode relict, located in the maximum density gradient and propagating purely azimuthally in the direction of the electron diamagnetic drift. The frequency of this drift wave mode $(f=4.1 \mathrm{kHz})$ is in good agreement with the Hasegawa-Mima drift wave dispersion relation. Along the radial density profile the intermittency of density fluctuations increases as characterized by positive high amplitude density bursts $(\tilde{n} / \sigma \sim 7)$ and non-Gaussian probability distribution functions (maximum values skewness $S=2.9$ and kurtosis $K=11$ ). The peak burst density in the plasma edge corresponds to the density fluctuation amplitude in the maximum radial density gradient region. This is seen as a strong indication that the density bursts in the edge plasma have their origin in the radial density gradient.

The spatiotemporal conditional average analysis of density fluctuations (Figs. 7 and 8) reveals that the high amplitude density bursts in the plasma edge are connected with coherent structures. They peel-off an $m=1$ drift wave mode and propagate mainly azimuthally in the direction of the $\mathrm{E} \times \mathrm{B}$-velocity (opposite to the drift mode propagation direction), but also has a strong radial velocity component. The values of the azimuthal and radial velocities are $v_{\text {pol }}=760 \mathrm{~m} / \mathrm{s}$ and $v_{\text {rad }}=230 \mathrm{~m} / \mathrm{s}$, respectively. This radial velocity corresponds to approximately $10 \%$ of the ion sound speed, which is similar to what has been found previously in toroidal [2] and other linear devices [10]. In the plasma edge the amplitude of the structure strongly exceeds the time averaged density with $\tilde{n}_{\text {burst }} \approx 6 n_{0}$. The structure is azimuthally distorted having a maximum poloidal correlation length of $l_{\text {corr,pol }} \sim 4 \rho_{s}$, which is in rough agreement with the findings presented in Ref. [10].

The formation process of the turbulent structure seems to be closely linked to the primary drift wave instability. Measurements of the phase relation between plasma density and plasma potential fluctuations in the maximum density gradient (not shown here) revealed a phase shift of $\delta_{\tilde{n}, \tilde{\phi}} \sim \pi / 2$, which results in a radially outward directed fluctuation-induced particle flux $\Gamma=\tilde{n} \tilde{v} \sim k_{\theta} \tilde{n} \tilde{\phi} \sin \delta_{\tilde{n}, \tilde{\phi}}$. The azimuthal electric field at the position of the density structures, given by the phase shift $\delta_{\tilde{n}, \tilde{\phi}}$, results in a radial $\mathrm{E} \times \mathrm{B}$-drift. Thus, the 
structure propagation is likely to be caused by advection in the self-consistent potential distributions. The quantitative analysis of radial structure propagation speed and the associated potential will be subject of future investigations. First comparison of the experimental findings presented here with the neutral wind model [12] suggests consistency: Due to the different heating rates of neutrals in the core plasma and in the edge plasma by elastic ion-neutral collisions, a net force $F_{i n}$ acts on the structures and points radially outwards. Inserting the experimentally observed peak density of turbulent structure and temperatures of $T_{0}^{\text {core }}=0.05 \mathrm{eV}$ and $T_{0}^{\text {edge }}=0.02 \mathrm{eV}$ for the neutral argon atoms in the core plasma and in the edge plasma, respectively, we obtain from the neutral wind model a radial structure velocity of $v_{n w}=272 \mathrm{~km} / \mathrm{s}$ of the turbulent structure, which is in good agreement with the experimental findings. However, the estimated radial velocity obtained from the neutral wind model sensitively depends on the neutral temperatures, which will be measured in detail by laser induced fluorescence (LIF) to validate the above assumptions. The discussed mechanisms causing the radial propagation of turbulent structures in the plasma edge (selfadvection and neutral wind) may happen at the same time. Which process is the dominant mechanism will be the subject of further investigations. 
[1] J. L. Terry, S. J. Zweben, K. Hallatschek, B. LaBombard, R. J. Maqueda, B. Bai, C. J. Boswell, M. Greenwald, D. Kopon, W. M. Nevins, et al., Phys. Plasmas 10, 1739 (2003).

[2] J. A. Boedo, D. L. Rudakov, R. A. Moyer, G. R. McKee, R. J. Colchin, M. J. Schaffer, P. G. Stangeby, W. P. West, S. L. Allen, T. E. Evans, et al., Phys. Plasmas 10, 1670 (2003).

[3] S. J. Zweben, R. J. Maqueda, D. P. Stotler, A. Keese, J. Boedo, C. E. Bush, S. M. Kaye, B. LeBlanc, J. L. Lowrance, V. J. Mastrocola, et al., Nucl. Fusion 44, 134 (2004).

[4] B. LaBombard, M. V. Umansky, R. L. Boivin, J. A. Goetz, J. Hughes, B. Lipschultz, D. Mossessian, C. S. Pitcher, and J. L. Terry, Nucl. Fusion 40, 2041 (2000).

[5] O. Grulke, J. L. Terry, B. LaBombard, and S. J. Zweben, Phys. Plasmas 13, 012306 (2006).

[6] J. A. Boedo, D. Rudakov, R. Moyer, S. Krasheninnikov, D. Whyte, G. McKee, G. Tynan, M. Schaffer, P. Stangeby, P. West, et al., Phys. Plasmas 8, 4826 (2001).

[7] S. I. Krasheninnikov, Phys. Lett. A 283, 368 (2001).

[8] O. E. Garcia, V. Naulin, A. H. Nielsen, and J. J. Rasmussen, Phys. Rev. Lett. 92, 165003 (2004).

[9] G. Y. Antar, Phys. Plasmas 10, 3629 (2003).

[10] T. A. Carter, Phys. Plasmas 13, 010701 (2006).

[11] G. Y. Antar, Contrib. Plasma Phys. 44, 217 (2004).

[12] S. I. Krasheninnikov and A. I. Smolyakov, Phys. Plasmas 10, 3020 (2003).

[13] R. W. Boswell, Plasma Phys. Controlled Fusion 26, 1147 (1984).

[14] C. M. Franck, O. Grulke, and T. Klinger, Phys. Plasmas 10, 323 (2003).

[15] I. D. Sudit and F. F. Chen, Plasma Sources Sci. Technol. 3, 162 (1994).

[16] C. Schröder, O. Grulke, T. Klinger, and V. Naulin, Phys. Plasmas 12, 042103 (2005).

[17] R. J. Adrian, Phys. Fluids 22, 2065 (1979).

[18] T. Huld, A. H. Nielsen, H. L. Pécseli, and J. J. Rasmussen, Phys. Fluids B 3, 1609 (1991).

[19] A. Hasegawa and K. Mima, Phys. Rev. Lett. 39, 205 (1976). 